Antony N. Thomas MB BS FFARCS, Brian J. Pollard MB ChB BPharm FFARCS

\title{
Renal transplantation and diabetic autonomic neuropathy
}

This report describes six episodes of cardiovascular collapse in the perioperative period of a young diabetic woman undergoing general anaesthesia for renal transplantation and a similar episode after a second anaesthetic. She was subsequently found to have an autonomic neuropathy. Recommendations for the management of similar patients are made.

Autonomic neuropathy is a recognised complication of diabetes mellitus. Sudden and unexpected cardiorespiratory collapse has previously been described in patients with this complication. Page ${ }^{1}$ described 12 episodes of cardiorespiratory or respiratory collapse in eight young diabetic patients. In three of these, apnoea occurred during anaesthesia and in a further two, cardiorespiratory collapse occurred immediately after anaesthesia. Ciccarelli ${ }^{2}$ described a similar episode of severe hypotension and bradycardia in a patient with renal failure who was undergoing general anaesthesia for the insertion of a peritoneal dialysis cannula. This report describes six episodes of cardiovascular collapse during the perioperative period of a patient undergoing general anaesthesia for cadaveric renal transplantation and a similar episode in the same patient after a subsequent anaesthetic.

\section{Case report}

A 36 -year-old, $60 \mathrm{~kg}$, female patient was admitted for cadaveric renal transplantation. She had been an insulindependent diabetic for 25 years and three years before admission had been diagnosed as having chronic renal failure secondary to diabetic nephropathy. She had required peritoneal dialysis for the last 20 months and she had had previous uneventful general anaesthetics.

\section{Key words}

COMPLICATIONS: cardiovascular, diabetes, neuropathyautonomic; HEART: arrhythmia, bradycardia; KIDNEY: failure.

Department of Anaesthesia, Manchester Royal Infirmary, Oxford Road, Manchester M13 9LW.

Address correspondence to: Dr. A. N. Thomas.
On examination, her heart rate was $80 \mathrm{bpm}$, her arterial pressure was $140 / 80 \mathrm{mmHg}$, and she had mild ankle oedema but no other signs of heart failure. She had a non-proliferative diabetic retinopathy. Ankle jerk reflexes were absent but she had normal vibration and position sense in her feet. Her preoperative investigations showed haemoglobin $8.7 \mathrm{~g} \cdot \mathrm{dl}^{-1}$, serum potassium $4.9 \mathrm{mmol} \cdot \mathrm{l}^{-1}$ sodium $135 \mathrm{mmol} \cdot \mathrm{l}^{-1}$ and creatinine $928 \mathrm{mmol} \cdot \mathrm{l}^{-1}$. The chest $x$-ray and ECG were within normal limits. The blood glucose was $6.5 \mathrm{mmol} \cdot \mathrm{1}^{-1}$ prior to induction of anaesthesia.

The patient was anaesthetised without premedication at 18:00 hr on the evening of admission. Anaesthesia was induced with morphine $10 \mathrm{mg}$, droperidol $2 \mathrm{mg}$, thiopentone $175 \mathrm{mg}$ and atracurium $40 \mathrm{mg}$. The trachea was intubated and anaesthesia was maintained using controlled ventilation with 33 per cent nitrous oxide in oxygen and isoflurane. Repeated boluses of atracurium 10 mg were given to maintain muscle relaxation. The ECG, arterial pressure and pulse were monitored from induction of anaesthesia; central venous pressure and neuromuscular transmission were measured at intervals and blood glucose checked every $30 \mathrm{~min}$.

The intraoperative period was unremarkable until the cardaveric kidney was revascularized. This resulted in a brisk haemorrhage. The patient's systolic arterial pressure decreased from 110 to $70 \mathrm{mmHg}$, with a simultaneous decrease in the heart rate from 80 to $35 \mathrm{bpm}$. The heart rate did not increase following $2 \mathrm{mg}$ atropine but did increase in response to fluid replacement of one unit of whole blood and one litre of polygeline (Haemaccel).

At the end of surgery neostigmine $2.5 \mathrm{mg}$ and glycopyrronium $0.5 \mathrm{mg}$ were given and when the train-of-four ratio was greater than 75 per cent, the trachea was extubated. The patient was awake, talking and oriented. Monitoring was discontinued and she was moved from the operating theatre to the recovery area. Shortly after this and before she could be given any oxygen she became unconscious, apnoeic and her pulse could not be palpated. External cardiac massage was commenced, a tracheal tube was inserted and the lungs were ventilated with 100 per cent oxygen. The pulse rapidly returned and the ECG monitor showed sinus rhythm at a rate of $80 \mathrm{bpm}$. She regained consciousness and the trachea was extubated within 10 
min. Her investigations at the time were unremarkable except for a metabolic acidosis ( $\mathrm{pH} 7.2$ ). She was given supplemental oxygen overnight during which time she passed over two litres of urine.

During the afternoon on the first postoperative day and the early hours of the second postoperative day the patient had a further four episodes of respiratory arrest and loss of consciousness. During the third and fourth of these, a profound nodal bradycardia was noted on the ECG. She responded rapidly to basic life support measures on each occasion. The patient was not receiving supplemental oxygen in the periods preceding the first three of these arrests. Biochemical investigations immediately after each episode were unremarkable except for a small persistent metabolic acidosis ( $\mathrm{pH} 7.3$ ).

Ten days after her original operation the patient was scheduled for a ureteric reimplantation under general anaesthesia. A similar anaesthetic technique was used to the earlier occasion and was uneventful. She received supplemental oxygen overnight. This was discontinued the following morning and that afternoon she again developed a bradycardic episode. She was resuscitated and a temporary transvenous pacemaker was inserted. Coronary angiography was performed which demonstrated normal coronary arteries. The left ventricular enddiastolic pressure was $12 \mathrm{mmHg}$, and there was slight anterior wall dyskinesia. These findings are compatible with a mild infiltrative diabetic cardiomyopathy.

Two weeks after the second operation, autonomic function tests were performed using a protocol similar to that described by Ewing and Clarke. ${ }^{3}$ The heart rate response to a standard $40 \mathrm{mmHg}$ Valsalva manoeuvre showed a Valsalva ratio of 0.09 (normal > 1.21). The heart rate was unchanged in response to sustained hand grip, head down tilt or exercise testing. There was a rise of 20 $\mathrm{mmHg}$ in systolic and diastolic blood pressure in response to standing although there was no heart rate response. The diastolic blood pressure increased $5 \mathrm{mmHg}$ in response to hand grip (normal > $16 \mathrm{mmHg}$ ) but did not respond to exercise testing.

\section{Discussion}

There are similarities between this and other case reports. The patient described by Ciccarelli ${ }^{2}$ and six of the eight patients described by Page ${ }^{1}$ were female. All the patients were of a similar age ( 31 to 41 years) and had longstanding diabetes with severe end organ damage, including diabetic retinopathy. Several of the patients had chronic renal failure. Two of the patients described by Page ${ }^{1}$ collapsed in the immediate postoperative period; these and a further two of the eight patients he described may well have been hypoxic. Hypoxia would have been a most unlikely cause of bradycardia in the patient described by
Ciccarelli, ${ }^{2}$ or in our patient certainly on the first occasion and possibly on others. Thus, although important, hypoxia may not be the only cause of these bradycardic episodes.

The initial intraoperative bradycardia occurred during a period of sudden hypovolaemia. Bradycardia as a response to relative ${ }^{4}$ or absolute hypovolaemia $a^{5}$ is well documented and may be due to the intrinsic characteristics of the myocardial pacemaker cells. ${ }^{6}$ It is possible that this was the cause of our patient's initial bradycardia. The second episode occurred in the immediate postoperative period, at a time when patients are known to be at risk from hypoxia. ${ }^{7}$ The subsequent episodes of cardiorespiratory collapse occurred over the following $36 \mathrm{hr}$, and again $24 \mathrm{hr}$ after a subsequent anaesthetic. The ECG was being monitored during three of these episodes and demonstrated a profound nodal bradycardia on each occasion.

In retrospect, it seems likely that our patient was receiving supplemental oxygen preceding only the last of the postoperative collapses which she experienced after the first operation. A reduction in functional residual capacity lasts several days after abdominal surgery ${ }^{8}$ and this, together with a co-existing anaemia, may well have rendered our patient at least periodically hypoxaemic. Hypoxia results in a faster rate of ventricular failure ${ }^{9}$ and a slower rate of recovery in the diabetic hear ${ }^{10}$ and this susceptibility to hypoxia without a normal sympathetic drive may offer an explanation for some of the episodes of bradycardia and collapse. The negative inotropic effects of hypoxia would have been compounded by the presence of a metabolic acidosis. It is notable that none of these episodes occurred on the two postoperative nights when added oxygen was administered.

A Valsalva Ratio of 0.09 and an absent heart rate response to standing are indicative of parasympathetic damage. ${ }^{3}$ The degree of sympathetic damage is less obvious as there was a normal increase in blood pressure in response to standing, although the increase in diastolic blood pressure in response to hand grip was abnormally small. A diagnosis of mild infiltrative diabetic cardiomyopathy was made both on the clinical history and on the appearance of the left ventricle during angiography. The original decision to perform coronary angiography had been taken because of the strong association of ischaemic heart disease with diabetes and chronic renal failure and it was felt that the patient may have had a stenotic lesion that may have been amenable to angioplasty. In spite of a long history of diabetes and chronic renal failure coronary angiography was normal.

In summary, although diabetic autonomic neuropathy may not be obvious from the clinical history and examination, it should be suspected where there is a long diabetic history with evidence of multiple end organ damage. The 
diagnosis can be confirmed by simple non-invasive investigations. Patients with this condition are prone to episodes of profound bradycardia which may often be caused by hypoxia. It is recommended that they should have their ECG and oxygen saturation monitored and that they should be given added oxygen until at least the end of the second postoperative night after renal transplantation. In addition, there may be a place for temporary demand pacing in patients known to be susceptible to this condition, to cover the perioperative period during major surgery.

\section{Acknowledgements}

We thank Mr. R.W.G. Johnson for permission to report his patient, Dr. Hugh Love for permission to report the results of the cardiological investigations and the help and advice given by both.

\section{References}

1 Page MMcB, Watkins PJ. Cardiorespiratory arrest and diabetic autonomic neuropathy. Lancet 1978; 1: 14-6.

2 Ciccarelli $L L$, Ford $C M, T$ sueda $K$. Autonomic neuropathy in a patient with renal failure. Anesthesiology 1986; 64: 283-7

3 Ewing DJ, Clarke B.F. Diagnosis and management of diabetic autonomic neuropathy: Br Med J 1982; 285: 916-8.

4 Greene NM. The cardiovascular system. Physiology of spinal anaesthesia. Baltimore, London. Williams \& Wilkins 1981; 63-133.

5 Secher NH. Bradycardia during reversible haemorrhage shock - a forgotten observation? Clinical Physiology 1985; 5: 315-23.

6 Pathak $C L$. The fallacy of the Bainbridge Reflex. Am Heart J 1966; 72: 577.

7 Meiklejohn BH, Smith G, Elling AE, Hindoona N. Arterial oxygen desaturation during post-operative transporation: the influence of operation site. Anaesthesia 1987; 42: 1313-5.

8 Craig $D B$. Post-operative recovery of pulmonary function. Anaesth Analg 1981; 60: 46-52.

9 Feuvray $D$, Idell-Wenger $J A, N e e l y S R$. Effects of ischaemia on rat myocardial function and metabolism in diabetes. Circ Res 1979; 44: 922-33.

10 Hearse DJ, Stewart DA, Chain EB. Diabetes and the survival and recovery of the anoxic myocardium. J Mol Cell Cardiol. 1975; 7: 397-415.

\section{Résumé}

Nous avons observé six épisodes de collapsus cardio-vasculaire en péri-opératoire de transplantation rénale sous anesthesie générale chez une jeune diabétique. Elle encourut le même probleme après une deuxième anesthèsie et on diagnostiqua chez elle une dysfonction neuro-végétative. Nous revoyons les critères diagnostiques de ce type de neuropathie et la conduite d tenir. 